\title{
One-particle spectral densities and phase diagrams of one-dimensional proton conductors
}

\author{
R. Ya. Stetsiv/* \\ Institute for Condensed Matter Physics of the National Academy of Sciences of Ukraine, \\ 1 Svientsitskii St., 79011 Lviv, Ukraine
}

Received November 19, 2020, in final form April 2, 2021

\begin{abstract}
The equilibrium states of one-dimensional proton conductors in the systems with hydrogen bonds are investigated. Our extended hard-core boson lattice model includes short-range interactions between hydrogen ions, their transfer along the hydrogen bonds with two-minima local anharmonic potential, as well as their interbond hopping, and the modulating field is taken into account. The exact diagonalization method for finite onedimensional system with periodic boundary conditions is used. The existence of various phases of the system at $T=0$, depending on the values of short-range interactions between particles and the modulating field strength, is established by analyzing the character of the obtained frequency dependence of one-particle spectral density; the phase diagrams are built.
\end{abstract}

Key words: proton conductor, hard-core boson model, spectral density, phase diagrams

\section{Introduction}

Ionic conductors are known since the times of Faraday [1], but the biggest urge for the development of this direction in solid state physics was the discovery in 1960th of the structures with charge carriers that are ions of silver $\left(\alpha-\mathrm{AgI}, \mathrm{Ag}_{2} \mathrm{~S}\right)$. These crystals are characterised by the presence of high temperature phase with high ionic conductivity. This phase afterwards was referred to as superionic (the same as crystals). The further research resulted in the opening of a new class of the systems where charge carriers are hydrogen ions. They are ferroelectric or ferroelastic crystals at low temperatures, but at higher temperatures they undergo transition to superprotonic phase, while the conductivity is increased by several orders of magnitude (among others there are compounds of the general form $\mathrm{MeHXO}_{4}$, where $\mathrm{Me}=\mathrm{Cs}, \mathrm{Rb}, \mathrm{NH}_{4} ; \mathrm{X}=\mathrm{S}, \mathrm{Se}$ ). Numerous structural studies have shown that in low-temperature phase the protons are clearly in the fixed positions, while in high-temperature phase they are distributed with equal probability between multiple positions in the unit cell. Much effort is presently put for the synthesis of new structures with high ionic conductivity. This is predetermined by their use in the areas of new technique such as hydrogen energetics, chemical sources of current, electronics, control and measurable devices for special purposes. As an example, we can cite a series of lithium conductive materials synthesized from perovskite structures $\mathrm{La}_{23-x} \mathrm{Li}_{3 x} \mathrm{TiO}_{3}$-type [2-4]. The superionic state is educed, for example, in $\mathrm{Ag}_{2} \mathrm{~S}$, $\mathrm{AgI}, \mathrm{AgBr}, \mathrm{CuBr}, \mathrm{Cu}_{2} \mathrm{~S}, \mathrm{CuCl}, \mathrm{RbAg}_{4} \mathrm{I}_{5}$ compounds where the metallic cation migrates. Structures with the large concentration of admixture ions: oxidizing solid solutions $\mathrm{MO}_{2}-\mathrm{M}_{2}^{\prime} \mathrm{O}_{3}$ and $\mathrm{MO}_{2}-\mathrm{M}^{\prime \prime} \mathrm{O}$ type, where $\mathrm{M}-\mathrm{Zr}, \mathrm{Ge} ; \mathrm{M}^{\prime}-\mathrm{Ca}, \mathrm{Sr}, \mathrm{Ba} ; \mathrm{M}^{\prime \prime}-\mathrm{S}$, $\mathrm{Y}$; structures of $\mathrm{Na}_{2} \mathrm{O} \cdot 11 \mathrm{Al}_{2} \mathrm{O}_{3}$-type (Na migrates in planes between $\mathrm{Al}_{2} \mathrm{O}_{3}$ blocks). Without regard to great successes, only a few substances with high ionic conductivity and stable against chemical and mechanical action and possessing other specific properties are presently known. The conductivity of ionic conductors is particularly high when the number of ions is much less than the number of positions in a lattice, i.e., when there are vacancies. Therefore, a lot of free positions facilitate the probability of ion hopping from one position to another. In particular, in the

* stetsiv@icmp.lviv.ua 
superionic phase of $\mathrm{AgI}\left(T>147^{\circ} \mathrm{C}\right), 2$ conductivity ions of $\mathrm{Ag}^{+}$are statistically distributed between 42 settled positions of different type in the unit cell.

Models of a lattice gas type with Pauli statistics of particles are often used for the description of ionic conductors [5-20]. Here, particles are of Bose nature but they also obey the Fermi rule. The lattice model of Pauli particles is similar to the Bose-Hubbard model in the hard-core approximation (provided that the occupation numbers are restricted, $n_{i}=0.1$ ). Such a lattice model can describe the emergence of a superfluid (SF)-type state even in the absence of a direct interaction between particles [6] 8].

One-dimensional ion conductors were investigated in our previous work [8]. The existence of various phases of the system, depending on the values of interactions between particles $V$ and modulating field strength $A$, was established. In this work we examine finite one-dimensional ionic conductors with the two-minima local potential for ions. Usually, they are proton conductors in the systems with hydrogen bonds. Ab initio studies of many researchers show that in the systems with hydrogen bonds, there remains $0.75-0.8$ of electron $(0.75 e-0.8 e)$ at the hydrogen ion. Despite this, it is generally accepted to call conductors with hydrogen bonds proton conductors. We have got not a proton but an ion of hydrogen having a charge $+0.2 \div+0.25$. Thus, we cannot state that these are Fermi-particles or Bose-particles, so we use hard-core boson lattice model. Our extended hard-core boson lattice model includes short-range interactions between ions, their transfer along hydrogen bonds with the two-minima local anharmonic potential as well as their inter-bond hopping, and modulating field is taken into account. Here, unlike the previous case, we have two different interactions between ions ( $V$ on the hydrogen bond, and $w$ between bonds) and consequently two different transfers. An exact diagonalization method for a finite one-dimensional system with periodic boundary conditions is used. Energy spectrum and one-particle spectral densities are calculated; the phase diagrams are built.

\section{The model}

We use the lattice gas quantum model for the description of ionic (proton) conductors. This model corresponds to the hard-core boson limit, if the particles obey the Pauli statistics. If the positions of particles are characterized by symmetric local potential possessing two minima on the bond, the two stage nature of particle transport is taken into account via two constants of particle transfer $\left(\Omega_{0}\right.$ for intra-bond hopping between two positions $a$ and $b$ on each bond as well as $\Omega_{R}$ for inter-bond transfer between hydrogen bonds that arises due to orientational motion of ionic groups). That is why the model is referred to as orientational-tunneling model [21]. It also includes the correlation between nearest ions caused by the short-range repulsion (the corresponding energies parameters are: $V$ on the bond and $w$ for the neighbor bonds); the modulating field is also included (the parameter $A$ ). The field $A$ causes a spatial modulation of the ion distribution in the so-called ordered phase (the existence of such a phase at low temperatures is a characteristic feature of superionic conductors).

The Hamiltonian of this model for the chain-like structure can be presented as follows:

$$
\begin{aligned}
H & =-\mu \sum_{i}\left(n_{i a}+n_{i b}\right)+V \sum_{i} n_{i a} n_{i b}+w \sum_{i} n_{i b} n_{i+1, a}+\Omega_{0} \sum_{i}\left(c_{i a}^{+} c_{i b}+c_{i b}^{+} c_{i a}\right) \\
& +\Omega_{R} \sum_{i}\left(c_{i b}^{+} c_{i+1, a}+c_{i+1, a}^{+} c_{i b}\right)+A \sum_{i}\left(n_{i b}-n_{i a}\right) .
\end{aligned}
$$

Here, operators $c_{i, \alpha}\left(c_{i, \alpha}^{+}\right)$are the hard-core boson (HCB) operators. They describe the process of annihilation (creation) of particle on bond $i$ in position $\alpha(\alpha=a, b)$; therefore, $n_{i, \alpha}=c_{i, \alpha}^{+} c_{i, \alpha}$ is the occupation number of particles in this position (here, the eigenvalues of $n_{i, a}$ and $n_{i, b}$ are equal to 0 or 1 ). At different sites, HCB creation and annihilation operators commute as usual of bosons:

$$
\left[c_{k}, c_{m}^{+}\right]=\left[c_{k}, c_{m}\right]=\left[c_{k}^{+}, c_{m}^{+}\right]=0, \quad k \neq m
$$

At the same site these operators satisfy anticommutation relations typical of fermions:

$$
\left\{c_{m}, c_{m}^{+}\right\}=1, \quad c_{m}^{+2}=c_{m}^{2}=0 .
$$


The energy spectrum of finite one-dimensional system with periodic boundary conditions is calculated using the exact diagonalization method. The Hamiltonian matrix as well as $c_{i, a}$ and $c_{i, a}^{+}$matrices are constructed on the basis of many-particle states $\left|n_{1, a} n_{1, b} \ldots n_{N, a} n_{N, b}\right\rangle$. The Hamiltonian matrix on the basis of these states is diagonalized numerically. Such an operation corresponds to the transformation

$$
U^{-1} H U=\widetilde{H}=\sum_{p} \lambda_{p} \widetilde{X}^{p p},
$$

where $\lambda_{p}$ are eigenvalues of the Hamiltonian, $\widetilde{X}^{p p}$ are Hubbard operators (in general, $\widetilde{X}^{p q}=|p\rangle\langle q|$ ), (see [22], also [23]). The creation and annihilation operators are presented in the form

$$
U^{-1} c_{i, \alpha} U=\sum_{p q} A_{p q}^{i, \alpha} \widetilde{X}^{p q}, \quad U^{-1} c_{i, \alpha}^{+} U=\sum_{r s} A_{r s}^{i, \alpha *} \widetilde{X}^{s r},
$$

where coefficients $A_{p q}^{i, \alpha}$ are the matrix elements of operator $c_{i, \alpha}$ on the new bases.

Like in our previous work [8], we construct two-time temperature Green's functions $G_{i, \alpha ; i, \alpha}=$ $\left\langle\left\langle c_{i, \alpha} \mid c_{i, \alpha}^{+}\right\rangle\right\rangle$containing the information about the one-particle spectrum of the system. We introduce Green's functions of two types, namely, the commutator Green's function

$$
\left\langle\left\langle c_{i, \alpha}(t) \mid c_{i, \alpha}^{+}\left(t^{\prime}\right)\right\rangle\right\rangle^{(c)}=-\mathrm{i} \Theta\left(t-t^{\prime}\right)\left\langle\left[c_{i, \alpha}(t), c_{i, \alpha}^{+}\left(t^{\prime}\right)\right]\right\rangle
$$

and the anticommutator Green's function

$$
\left\langle\left\langle c_{i, \alpha}(t) \mid c_{i, \alpha}^{+}\left(t^{\prime}\right)\right\rangle\right\rangle^{(a)}=-\mathrm{i} \Theta\left(t-t^{\prime}\right)\left\langle\left\{c_{i, \alpha}(t), c_{i, \alpha}^{+}\left(t^{\prime}\right)\right\}\right\rangle .
$$

One-particle spectral densities are determined by the imaginary parts of those Green's functions

$$
\begin{aligned}
\rho(\omega) & =-\frac{1}{\pi N} \sum_{j=1}^{N} \sum_{\alpha} \operatorname{Im}\left\langle\left\langle c_{j, \alpha} \mid c_{j, \alpha}^{+}\right\rangle\right\rangle_{\omega+\mathrm{i} \varepsilon} \\
& =-\frac{1}{\pi N} \sum_{j=1}^{N} \sum_{\alpha} \operatorname{Im}\left[\frac{1}{Z} \sum_{p q} A_{p q}^{j, \alpha} A_{p q}^{j, \alpha *} \frac{\mathrm{e}^{-\beta \lambda_{p}}-\eta \mathrm{e}^{-\beta \lambda_{q}}}{\omega-\frac{1}{\hbar}\left(\lambda_{q}-\lambda_{p}\right)+\mathrm{i} \varepsilon}\right] .
\end{aligned}
$$

Here, $Z=\sum_{p} \mathrm{e}^{-\beta \lambda_{p}}$.

We obtain spectral densities of commutator Green's function (2.6), when $\eta=1$, and anticommutator Green's function (2.7), when $\eta=-1$. Spectral densities have a discrete structure that includes a number of $\delta$-peaks owing to the finite chain size. If the chain size (i.e., the number of sites $N$ ) increases, the $\delta$-peaks are located more densely and, at $N \rightarrow \infty$, they form a band structure. We confined ourselves to the case $N=12$. We introduce the small parameter $\Delta$ to broaden the $\delta$-peaks in accordance with Lorentz distribution $\delta(\hbar \omega) \rightarrow \frac{1}{\pi} \frac{\Delta}{(\hbar \omega)^{2}+\Delta^{2}}$.

\section{One-particle spectral densities and phase diagrams}

In this work all calculations are performed for the temperature equal to zero $(T=0)$. Numerical values of all energy parameters (including $\hbar \omega$ ) are presented in units of parameter $\Omega_{0}$, and it is dimensionless. Experimental data, quantum-chemical calculations, semiempiric theoretical estimations offer a wide region of values of interaction between ions, $V=3 \cdot 10^{3} \ldots 10^{4} \mathrm{~cm}^{-1}, w=10^{3} \ldots 10^{4} \mathrm{~cm}^{-1}$, depending on the objects that are examined [24-26]. The ion transfer parameter $\Omega_{0}, \Omega_{R}$ can vary within wide limits, $40 \ldots 2500 \mathrm{~cm}^{-1}$. For example, it is obtained $V=5 \cdot 10^{3} \ldots 10^{4} \mathrm{~cm}^{-1}$ from the experimental data for $T_{c}$ in the case of $H$-bonded ferroelectrics. In our calculations we chose $\Omega_{R} / \Omega_{0}=0.5$. We changed the parameters of short-range interactions in wide limits: $V / \Omega_{0}=0,1, \ldots, 10, w / \Omega_{0}=0,1, \ldots, 10$. It is necessary to note that the problem is invariant in relation to simultaneous replacement of numerical values $w \leftrightarrow V, \Omega_{R} \leftrightarrow \Omega_{0}$. For convenience, we use the notation $\mu^{\prime}=\mu-(V+w) / 2$. 
One-particle spectral densities are calculated according to formula $(2.8)$. The existence of various phases of the system at $T=0$, depending on the values of interactions between particles and the modulating field strength, is established by analyzing the character of frequency dependence of oneparticle spectral density. According to works [27, 28], a characteristic feature of the commutator spectral density in the superfluid (SF) phase is the continuous continuation at $\omega=0$ of a negative branch (which exists at $\omega<0$ ) to a positive branch (which exists at $\omega>0$ ). The chemical potential of particles is located at the point $\omega=0$. In the charge ordering (CDW) phase, these branches are separated by the gap. The chemical potential of particles is located in the energy gap. We obtain a split of the spectrum into two subbands and the emergence of a modulated state. Charge-density-wave (CDW) state is the characteristic of the case of a half-filling of ionic sites $(\langle n\rangle=1 / 2)$ and one may observe the situation when all protons occupy " $a$ " positions (or all protons occupy " $b$ " positions) along the chain. This case corresponds to ferroelectric type ordering, though it has a more general meaning. The protons occupy only some of the positions available (while other positions remain unoccupied) which is a general feature of the ordered phases that exist in superionic crystals. We call this state CDW though the doubling of the lattice period is not observed. For the case of ionic conductor (with one minimum local potential for ions), the splitting of spectra occurred due to the charge ordering with the doubling of lattice period (see [8]). If one attempts to include the long-range interaction to our model, he or she will get the doubling of the lattice period also for proton conductor as well. At moving away from the half-filling, we get into the superfluid (SF) state. In this phase, the conductivity of the system grows by a few orders. Such a state experimentally looked like a superionic phase. As we go far away from half filling (for example, a decrease of chemical potential $\mu$ ) we get into a Mott insulator (MI) state. In this state, the commutator spectral density has no negative branch. The chemical potential is below the band and ions need some activation energy to induce their transport. MI state can also be observed when the chemical potential is located above the upper subband, and the commutator spectral density has only a negative branch. The average occupation number of the state at a given $\mu$ is calculated according to the spectral theorem, $\langle n\rangle=\int_{-\infty}^{\infty} \frac{\rho_{a}(\omega) \mathrm{d} \omega}{\mathrm{e}^{\beta \omega}+1}$, where $\rho_{a}$ is the anticommutator spectral density (the density of states).

In previous work [8], there was examined a chain with ten positions for ions $(N=10)$. For comparison, we repeated some previous calculations for the one dimensional systems with the one-minima local potential for the conductivity particles, though at $N=12$, in order to see the dependence of the results on $N$. The Hamiltonian of this problem is received from the Hamiltonian for the systems with two-well local anharmonic potential for ions (2.1), provided $V=w$ and $\Omega_{R}=\Omega_{0} \equiv t$. In figure 1 and figure 2 some diagrams of state at $N=12$ are compared with the ones obtained earlier in [8] at $N=10$. For the case, with the dependence of the phase borders on the value of interaction between particles $V$ shown in figure 1, which is a phase diagram in the $\left(\mu^{\prime}, V\right)$ coordinates, the numerical difference in the position

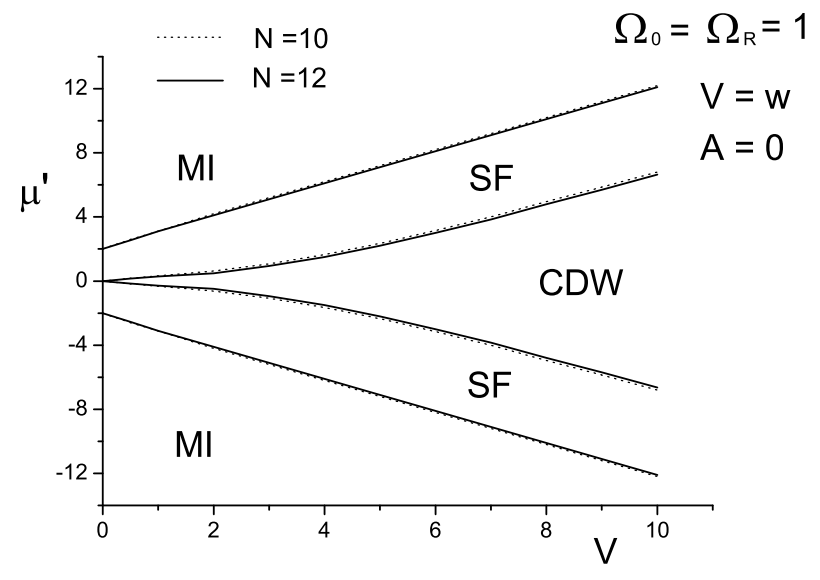

Figure 1. Phase diagram for a one-dimensional ionic conductor in the $\left(\mu^{\prime}, V\right)$ coordinates, $(T=0)$. 


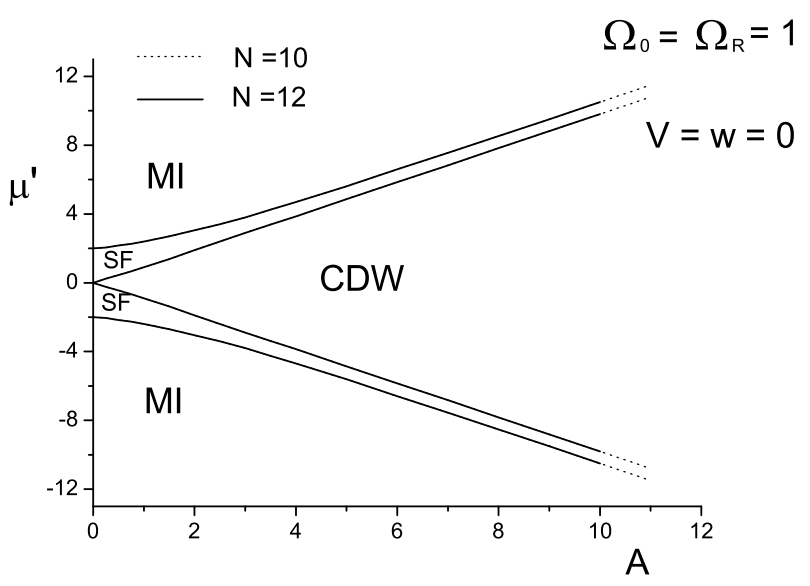

Figure 2. Phase diagram for a one-dimensional ionic conductor in the $\left(\mu^{\prime}, A\right)$ coordinates, $(T=0)$.

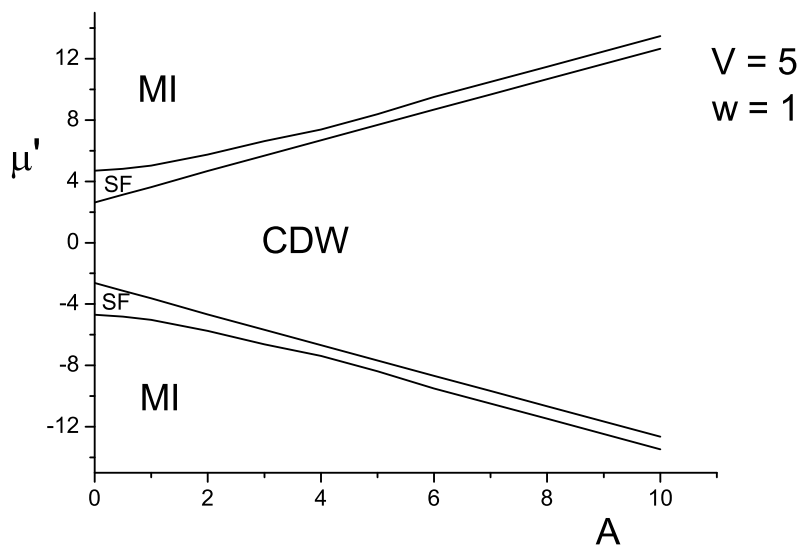

Figure 3. Phase diagram for a one-dimensional proton conductor in the $\left(\mu^{\prime}, A\right)$ coordinates, $(T=0)$.

of the phase-to-phase border (in the $\mu^{\prime}$ coordinate) between the cases of $N=10$ and $N=12$ is $2-3$ percent. For the case of phase diagram in the $\left(\mu^{\prime}, A\right)$ coordinates, (figure 2), this difference is 0.5 percent. The last one is built for the case $V=0$ and coincides with an exact diagram obtained analytically in the works $([11-14]$, see also [15]). An exact analytical solution can be obtained by applying the JordanWigner transformation, which makes it possible to pass from the Hamiltonian of hard-core bosons to the Hamiltonian of noninteracting spinless fermions (only in one-dimensional case). In this diagram, the lines separating the CDW and SF phases are linear on the field $A$ and look like $\mu^{\prime}=+A$ and $\mu^{\prime}=-A$ (see figure 2).

In this work we examine a finite one-dimensional ionic conductors with the two-minima local anharmonic potential for ions. Usually these are proton conductors in the systems with hydrogen bonds. All calculations are executed for $N=12$.

We obtained the phase diagrams of equilibrium states of the system depending on the interactions between ions and modulating field.

For example, the diagram of state showing the dependence on the modulating field $A$ is presented in figure 3 (here $V=5, w=1)$. It is a diagram in the $\left(\mu^{\prime}, A\right)$ coordinates. The line separating CDW and SF 


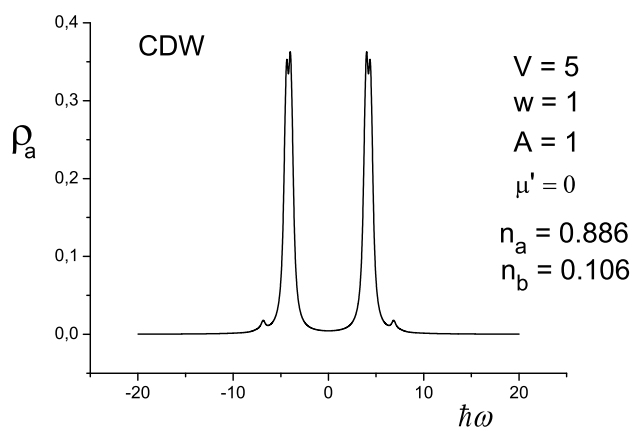

(a)

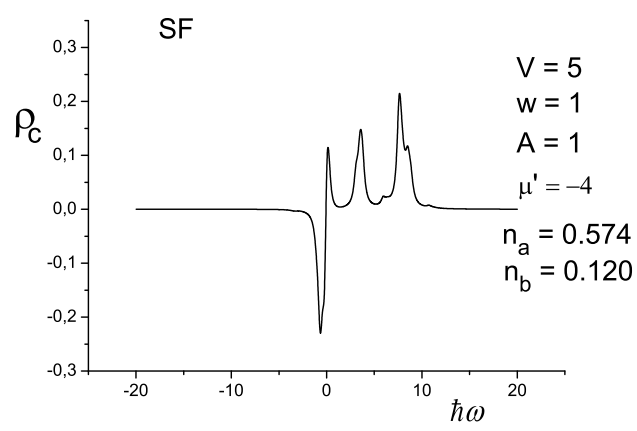

(c)

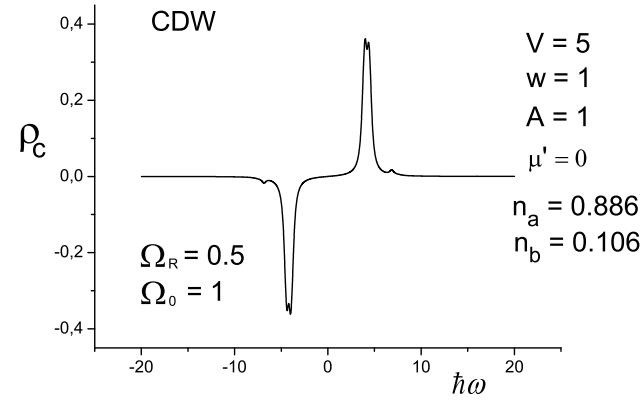

(b)

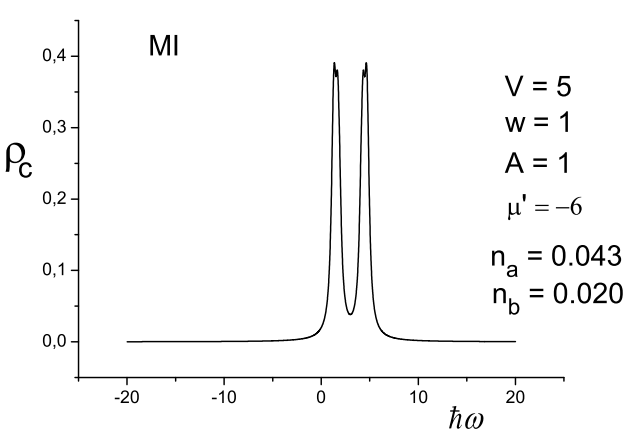

(d)

Figure 4. Anticommutator (a) and commutator (b-d) one-particle spectral density for various states of a one-dimensional proton conductor; $V=5, w=1, A=1, T=0, \Delta=0.25$. The chemical potential level is located at $\omega=0$.

phases in coordinates $\left(\mu^{\prime}, A\right)$ is a straight line depending on $A$ (see figure 3 ). The width of CDW phase (in $\mu^{\prime}$ coordinates) grows with an increase of the value of the modulating field.

Anticommutator and commutator one-particle spectral densities, that correspond to this diagram at $A=1$, are shown in figure 4 . We move from one phase to another by changing the chemical potential. The average occupation number of positions " $a$ " and " $b$ " in two-minima potential for each phase is presented in figure 4. For example, in CDW phase (at $A=1$ ), we obtained $n_{a}=0.886, n_{b}=0.106$, protons occupy mainly " $a$ " positions in two-minima local potential on the bond. The width of CDW phase is determined by the width of a gap in the energy spectrum.

Chemical potential level is placed at $\omega=0$. In CDW phase, chemical potential level is in the gap, while in SF phase we observe a continuous transformation of the negative branch of the commutator spectral density into the positive one at $\omega=0$.

In work [29] it is shown that an important characteristic of the SF phase is a divergence of the Fourier transform of the real part of commutator Green's function at zero frequency $(\omega=0)$ and zero wave vector $(k=0), \operatorname{Re} G_{k=0}(\omega=0) \rightarrow \infty$. In the case of the considered finite-chain model

$$
G_{k=0}(\omega=0)=\frac{1}{N} \sum_{i=1}^{N} \sum_{j=1}^{N}\left[\frac{1}{Z} \sum_{p q} A_{p q}^{i} A_{p q}^{j *} \frac{\mathrm{e}^{-\beta \lambda_{p}}-\mathrm{e}^{-\beta \lambda_{q}}}{\lambda_{p}-\lambda_{q}+\mathrm{i} \varepsilon}\right] .
$$

The static susceptibility $\operatorname{Re} G_{k=0}(\omega=0)$ calculated by us arrives at maximal values in SF phase, though even at $T=0$ those values remain finite, which is a result of the finite size of the chain and probably with unidimensionality of the object. $\operatorname{Re} G_{k=0}(\omega=0)$ at $V=5, w=1, A=1$ is shown in figure $5(T \neq 0)$. At $T=0$ (at the same parameters) we get a sharp peak in the region of SF phase, and susceptibility reaches the value of 1778.6, which is by three orders larger than at $T=0.1$ (see figure 5). At the further increase 


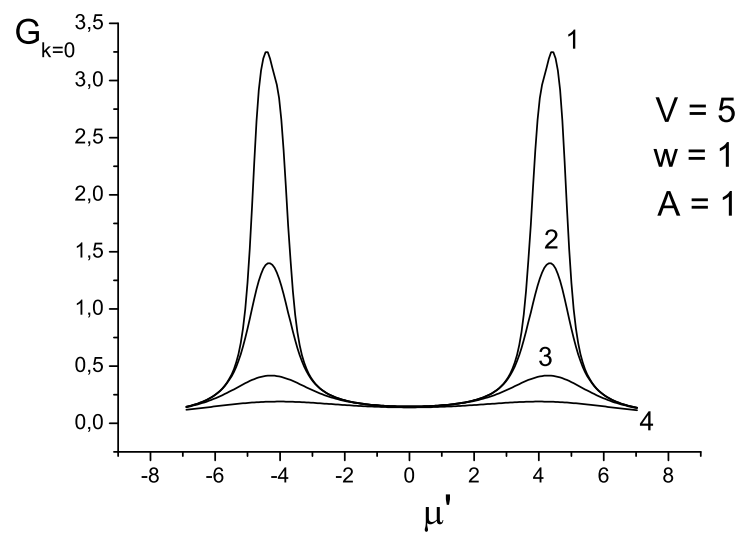

Figure 5. Fourier transform of the real part of commutator Green's function at zero frequency $(\omega=0)$ and zero wave vector $(k=0), \operatorname{Re} G_{k=0}(\omega=0): 1-T=0.1 ; 2-T=0.2 ; 3-T=0.5 ; 4-T=1$; $\Delta=1 \times 10^{-6}$.

of temperature, a maximum $\operatorname{Re} G_{k=0}(\omega=0)$ becomes smeared, which proves that in one-dimensional system a SF phase exists only at $T=0$. The regions of different phases at the above-mentioned parameters can be seen on the state diagram in figure 3

The CDW, SF and MI phases described above and the phase transitions between them for a onedimensional system exist only at zero temperature. At small temperatures, we can distinguish the regions of existence of the states of the CDW, SF and MI types as such in which the forms of the spectral functions
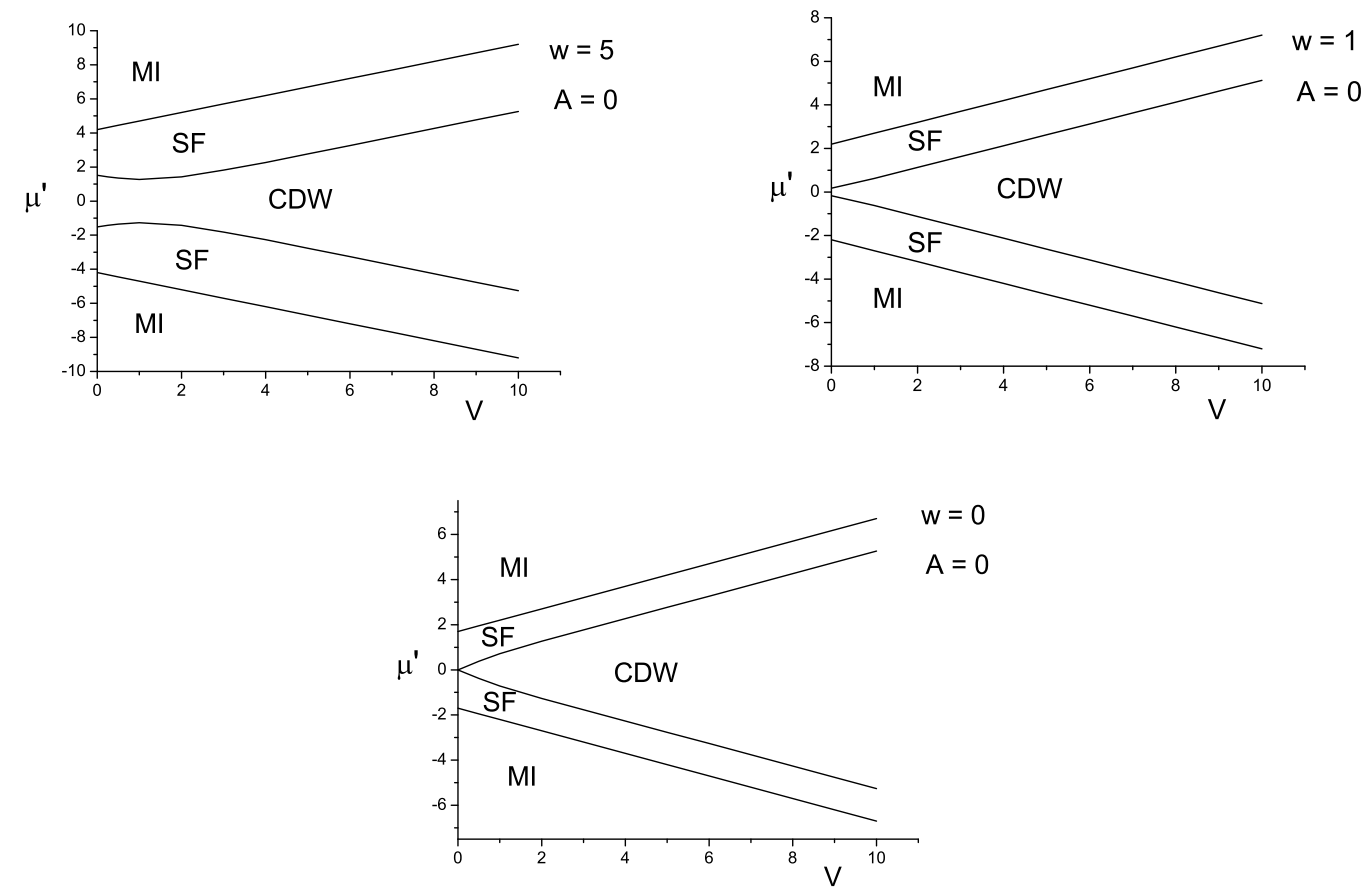

Figure 6. Phase diagrams for a one-dimensional proton conductor in the $\left(\mu^{\prime}, V\right)$ coordinates, $A=0$, $(T=0)$. 

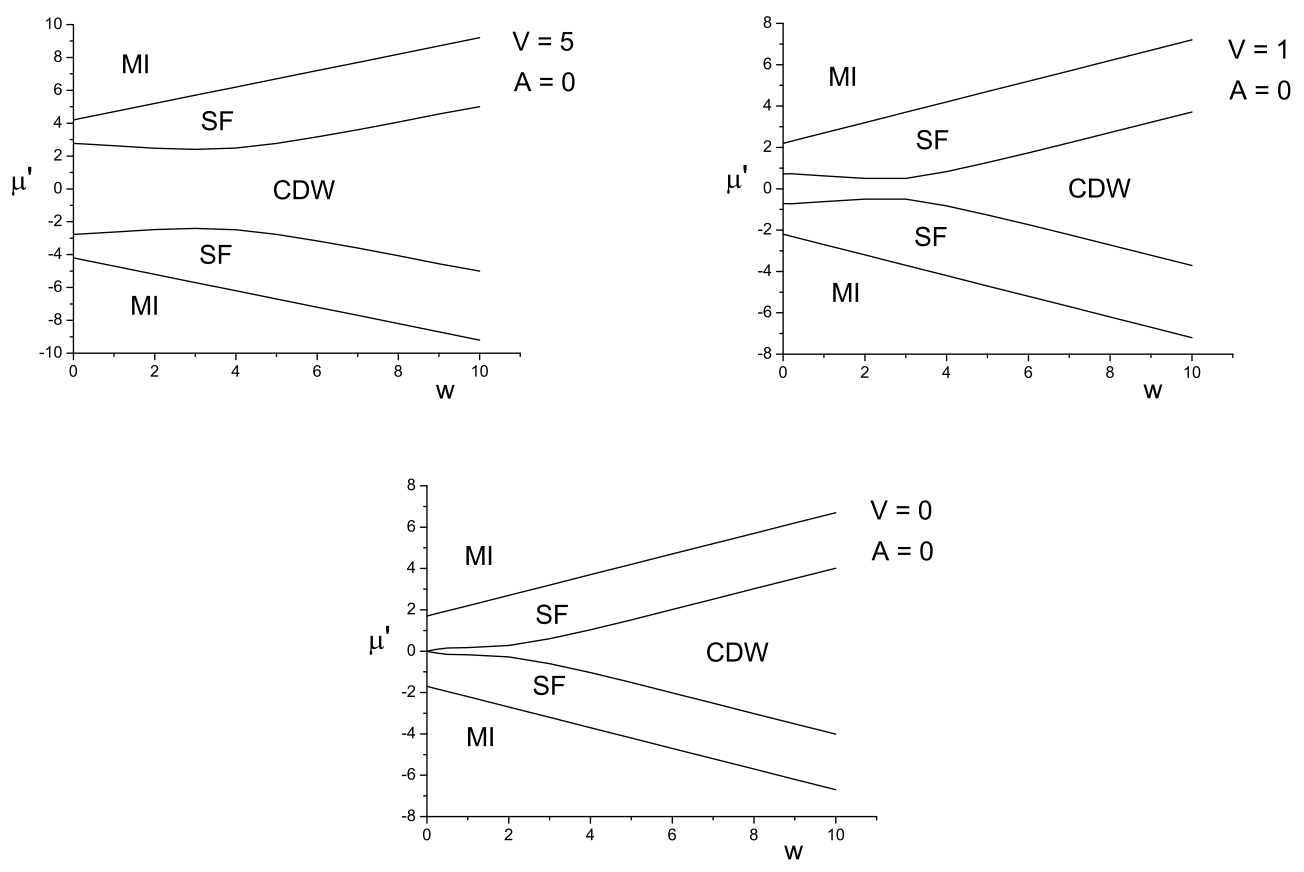

Figure 7. Phase diagrams for a one-dimensional proton conductor in the $\left(\mu^{\prime}, w\right)$ coordinates, $A=0$, $(T=0)$.

of those phases remain nearly the same as at $T=0$. In this case, the transition between the regions is not a genuine phase transition, but has a crossover character.

Phase diagrams of equilibrium states of the system depending on the short-range interactions between ions $V$ and $w$, obtained at $T=0, A=0$, are shown in figure 6 and figure 7 .

The characteristic features of all the latter diagrams is that the line separating SF and MI phases in $\left(\mu^{\prime}, V\right)$ and $\left(\mu^{\prime}, w\right)$ coordinates is strictly a straight line depending on $V$ or $w$. At large values of interactions $V$ and $w$ and at their further increase, we obtain the linear dependence of the width of CDW phase on the values of interactions between particles. At intermediate values of interactions there is a possibility of narrowing of CDW phase and the presence of a minimum of its width (in $\mu^{\prime}$ coordinates) depending on the values of interactions between ions.

\section{Conclusions}

The equilibrium states of one-dimensional proton conductors in the systems with hydrogen bonds are investigated. Our extended hard-core boson lattice model includes short-range interactions between ions (parameter $V$ on a hydrogen bond, and parameter $w$ between bonds), their transfer along hydrogen bonds with the two-minima local anharmonic potential as well as their inter-bond hopping, and the modulating field is taken into account. An exact diagonalization method for a finite one-dimensional system with periodic boundary conditions is used. The existence of various phases of the system at $T=0$ depending on the values of interactions between particles and the modulating field strength, is established by analyzing the character of the obtained frequency dependence of one-particle spectral density. It is shown that at $T=0$, the repulsive short-range interaction between particles $(V>0, w>0)$ results in the emergence of a gap in the energy spectrum in the limit of half-filling of ionic sites (the emergence of CDW phase). A similar effect also takes place under the influence of the modulating field $A$. Charge-density-wave (CDW) phase exists only at half-filling of ionic sites $\left(\left\langle n_{a}\right\rangle+\left\langle n_{b}\right\rangle\right) / 2=1 / 2$. Departing from the half-filling, we get into the SF phase. In this phase, the conductivity of the system 
grows by a few orders. The mentioned phases and phase transitions between them for the one-dimensional system exist only at $T=0$. The presence of SF phase is confirmed by the obtained sharp peak of the real part of static susceptibility $\operatorname{Re} G_{k=0}(\omega=0)$ at $T=0$. The predictable divergence of this susceptibility in the SF phase $\operatorname{Re} G_{k=0}(\omega=0) \rightarrow \infty$ was not reached by us which is predetermined by the finite size of the ion conductor and probably by its onedimensionality. However, it is shown that at small temperatures it is nearly by a few orders less than at $T=0$, and at the further increase of temperature a maximum $\operatorname{Re} G_{k=0}(\omega=0)$ becomes smeared which confirms that in onedimensional system a SF phase exists only at $T=0$.

We have obtained phase diagrams of equilibrium states of the system depending on the interactions between ions and the modulating field. It is shown that the width of CDW phase (in $\mu^{\prime}$ coordinates) grows with an increase of the value of the modulating field. The dependence of the width of CDW phase on short-range interactions between ions $V$ and $w$ is more complex. The characteristic feature of all the latter diagrams is that the line separating SF and MI phases in $\left(\mu^{\prime}, V\right)$ and $\left(\mu^{\prime}, w\right)$ coordinates is strictly a straight line depending on $V$ or $w$. At large values of interactions and at their further increase, we obtain a linear dependence of the width of CDW phase on the values of interactions between particles. At intermediate values of interactions, there is a possibility of narrowing of CDW phase and the presence of a minimum of its width (in $\mu^{\prime}$ coordinates) depending on the values of interactions between ions. We did not observe this kind of behavior for the ion conductor (with the one-minimum local potential for ions) (see [8]).

\section{References}

1. Faraday M., Philos. Trans. R. Soc. London, $1838,128,1$, doi:10.1098/rstl.1838.0009

2. Belous A., Solid State Ionics, 1996, 90, 193, doi 10.1016/S0167-2738(96)00406-7.

3. Belous A., J. J. Eur. Ceram. Soc., 2001, 21, 1797, doi:10.1016/S0955-2219(01)00118-2

4. Belous A., Ionics, 1998, 4, 360-363, doi:10.1007/BF02375878

5. Mahan G. D., Phys. Rev. B, 1976, 14, 780, doi:10.1103/PhysRevB.14.780

6. Stasyuk I. V., Dulepa I. R., Condens. Matter Phys., 2007, 10, 259, doi 10.5488/CMP.10.2.259

7. Stasyuk I. V., Dulepa I. R., J. Phys. Stud., 2009, 13, 2701, doi 10.30970/jps.13.2701

8. Stetsiv R. Ya., Stasyuk I. V., Vorobyov O., Ukr. J. Phys., 2014, 59, 515.

9. Micnas R., Ranninger J., Robaszkiewicz S., Rev. Mod. Phys., 1990, 62, 113, doi 10.1103/RevModPhys.62.113

10. Micnas R., Robaszkiewicz S., Phys. Rev. B, 1992, 45, 9900, doi 10.1103/PhysRevB.45.9900.

11. Rigol M., Muramatsu A., Olshanii M., Phys. Rev. A, 2006, 74, 053616, doi:10.1103/PhysRevA.74.053616

12. Rousseau V. G., Arovas D. P., Rigol M., Hebert F., Batrouni G. G., Scalettar R. T., Phys. Rev. B, 2006, 73, 174516, doi 10.1103/PhysRevB.73.174516

13. Rigol M., Muramatsu A., Phys. Rev. A, 2004, 70, 031603(R), doi 10.1103/PhysRevA.70.031603

14. Rigol M., Muramatsu A., Phys. Rev. A, 2005, 72, 013604, doi:10.1103/PhysRevA.72.013604.

15. Hen I., Iskin M., Rigol M., Phys. Rev. B, 2010, 81, 064503, doi $10.1103 /$ PhysRevB.81.064503.

16. Batrouni G. G., Scalettar R. T., Phys. Rev. B, 1992, 46, 9051, doi 10.1103/PhysRevB.46.9051

17. Batrouni G. G., Scalettar R. T., Phys. Rev. Lett., 2000, 84, 1599, doi $10.1103 /$ PhysRevLett.84.1599

18. Bernardet K., Batrouni G. G., Meunier J. L., Schmid G., Troyer M., Dorneich A., Phys. Rev. B, 2002, 65, 104519, doi 10.1103/PhysRevB.65.104519

19. Bernardet K., Batrouni G. G., Troyer M., Phys. Rev. B, 2002, 66, 054520, doi $10.1103 /$ PhysRevB.66.054520.

20. Schmid G., Todo S., Troyer M., Dorneich A., Phys. Rev. Lett., 2002, 88, 167208, doi $10.1103 /$ PhysRevLett.88.167208

21. Stasyuk I. V., Ivankiv O. L., Pavlenko N. I., J. Phys. Stud., 1997, 1, 418, doi:10.30970/jps.01.418

22. Hubbard J., Proc. R. Soc. London, Ser. A, 1965, 285, 542, doi 10.1098/rspa.1965.0124

23. Stasyuk I. V., Green's Functions in Quantum Statistics of Solid State: a Textbook, Ivan Franko National University of Lviv, Lviv, 2013, (in Ukrainian).

24. Münch W., Kreuer K. D., Traub U., Maier J., Solid State Ionics, 1995, 77, 10, doi $10.1016 / 0167-2738(95) 00045-8$

25. Hassan R., Campbell E. S., J. Chem. Phys., 1992, 97, 4362, doi $10.1063 / 1.463902$

26. Eckert M., Zundel G., J. Phys. Chem., 1988, 92, 7016, doi $10.1021 / \mathrm{j} 100335 \mathrm{a} 035$.

27. Menotti C., Trivedi N., Phys. Rev. B, 2008, 77, 235120, doi:10.1103/PhysRevB.77.235120.

28. Stasyuk I. V., Vorobyov O., Condens. Matter Phys., 2013, 16, 23005, doi:10.5488/CMP.16.23005

29. Stasyuk I. V., Mysakovych T. S., Condens. Matter Phys., 2009, 12, 539, doi:10.5488/CMP.12.4.539 


\title{
Одночастинкові спектральні густини і фазові діаграми одновимірних протонних провідників
}

\author{
Р. Я. Стеців \\ Інститут фізики конденсованих систем Національної академії наук України, \\ вул. Свєнціцького, 1, 79011 Львів, Україна
}

Ми досліджуємо рівноважні стани одновимірних протонних провідників в системах з водневими зв'язками. Наша розширена модель жорстких бозонів включає короткосяжну взаємодію між іонами водню, їх перенесення як вздовж водневих зв'язків з двомінімумним локальним ангармонічним потенціалом для протона, так і перенос частинок між сусідніми водневими зв'язками, а також модулююче поле. Розрахунки проведено методом точної діагоналізації для скінчених одновимірних систем з періодичними граничними умовами. Виходячи з характеру частотної залежності отриманих одночастинкових спектральних густин, встановлено існування різних фаз системи при $T=0$ в залежності від величини короткосяжної взаємодії між частинками і від величини модулюючого поля; побудовані фазові діаграми.

Ключові слова: протонний провідник, модель жорстких бозонів, спектральна густина, фазові діаграми 